\title{
REMARKS ON MODELING HOST-VIRAL DYNAMICS AND TREATMENT
}

\author{
JORGE X. VELASCO-HERNÁNDEZ*, JOSÉ A. GARCíA ${ }^{\dagger}$, AND \\ DENISE E. KIRSCHNER $\ddagger$
}

1. Introduction. Mathematical modeling of host-pathogen interactions is playing an increasingly important role in our understanding of the pathogenesis of infectious diseases. Many of the results have shaped our understanding of disease processes both at the population and cellular levels. In this tutorial we give an introduction to modeling the interaction of the human immune system with viral pathogens. Our collective research experience is focused on HIV so we use that as our prime example to explore approaches to understanding host-pathogen interactions and the treatment of disease. We present basic principles on the interaction of the immune system in response to the diverse set of pathogens that challenge humans. We then present a brief review of models for host-pathogen interactions at the cellular level, a summary of modeling HIV disease progression and subsequent chemotherapy, and finally, we present a model for the treatment of AIDS using the novel approach of robust control theory.

2. Host-pathogen interactions. The immune response represents a complex defense system against invading pathogens. Its main features involve induction, specificity and memory. Most organisms posses a nonspecific procedure for protection against pathogens: an innate response composed of physical and chemical barriers. Higher organisms also maintain an adaptive response comprised by humoral and cellular components that are pathogen specific (see [1]).

The most popular paradigm in immunology is that the key role of the immune response is to distinguish between self and non-self. This involves immune cells known as lymphocytes, that during their development, are elimintated if they recognize self [2]. Thus, immune cells that participate in the immune response recognize non-self molecules and can initiate an immune response cascade.

A second paradigm postulates that adaptive immune cells only respond if the antigen elicits different types and levels of tissue damage, not based on the nature of the antigen molecule $[3,4]$. This model considers the immune response as a defense system against pathogens.

When a foreign pathogen invades, an immune response is triggered. The innate elements attempt to suppress the invader, and days later the

\footnotetext{
*Departamento de Matemáticas, UAM Iztapalapa, México.

$\dagger$ Departamento de Investigación, Universidad La Salle, México.

${ }^{\ddagger}$ Department of Microbiology and Immunology, University of Michigan, Ann Arbor, MI 48109-0620, USA.
} 
adaptive immune components are developed. As the response progresses, the pathogen attempts to survive by various evasion mechanisms. Thus, a series of complex interactions between the host and the parasite are established.

2.1. Immune response to viral pathogens. Unlike bacteria, viruses must infect host cells to survive and replicate, and thus by definition, are intracellular microbes. Once virus binds to the host cell, the virus introduces its genetic material into the cell nucleus, and it remains there until the cell is activated and the viral genetic material is replicated. This process eventually kills the host cell. New virions are produced that are able to infect other cells.

When the invading virus circulates through the blood its receptor could be blocked by neutralizing antibodies. However, once a virus has already infected a cell, specific antibodies cannot reach it, and thus a cellular immune response is needed in order to suppress infection. Infected cells also are recognized by natural killer cells and then killed through the action of perforin and other molecules secreted by the NK cell. Both CTLs and NK cells are activated by TH1 cells through the action of cytokines.

Perhaps the main immune evasion mechanism of viruses is mutation. As the body is preparing to fight the virus by developing specific responses, the virus changes some of its antigenic proteins, via mutation, so that it is no longer recognized. Retroviruses (like the human immunodeficiency virus (HIV)) have a high spontaneous rate of mutation due to the enzyme called reverse transcriptase that does not possess a repair domain (exonuclease activity) which DNA polymerases usually have. This facilitates the mutation process. A second evasion mechanism comes by virtue of the intracellular lifestyle of viruses. Residing within host cells offers them protection from the immune response. Viruses have even developed ways to downregulate expression of host proteins on cells that signal infection.

2.2. Modeling host-viral interactions. Models of host-viral infections, namely HIV-host models, have a successful recent history. Many of the key results that have shaped our understanding of the T-cell and viral dynamics in HIV disease have come from mathematical modeling approaches [5-7]. Many others have given insight into HIV-immune dynamics as well as progression [8-26]. There is also a first attempt on modeling the interaction of cytokines and the HIV-infected immune system. This work examined the role of IL-2 immunotherapy in HIV-1 infection ([27]). Other viruses that have captured recent attention through modeling are Hepatitis $\mathrm{B}$ and $\mathrm{C}$ viruses $[28,29]$. Most viral-host models are developed using continuous and deterministic methods, and thus have been formulated using ordinary or partial differential equations. A few of these models e.g. [3034] are discrete and stochastic. The choice of modeling method is based on several considerations; e.g., the time frame of a study, the population sizes, and the types of questions posed. 
3. The human immunodeficiency virus. HIV has been recognized as the etiologic agent of the acquired immunodeficiency syndrome -AIDS ([35]. The United Nations estimates that there have been 8.4 million AIDS cases since the start of the global pandemic in the 1980s, with an estimated 30 million persons HIV-positive. Only 1.5 million cumulative cases have been officially reported by countries. An estimated 5 million adults and 1.5 million children have already died from AIDS. The impact of this emerging infectious disease is clear. Despite the impressive amount of research on this pathogen and the related areas of study, there are as yet no effective vaccines or treatment strategies that prevent the ultimate progression to AIDS and death. This is sentinel in realizing that many of the pathogenic mechanisms leading to disease progression are still unknown.

HIV is a retrovirus containing two single strands of RNA. It is an enveloped virus that carries its own reverse transcriptase for transcribing the viral RNA. HIV is transmitted via three primary routes: sexually, vertically (mother to child) and intravenously. Once infection takes place, the interaction of HIV and the immune system begins with $\mathrm{CD} 4^{+}$cells. The CD4 receptor appears mostly on helper $\mathrm{T}$ cells and some macrophages, and binds with the protein (ligand) gp120 (on the surface of the virus) initiating cellular infection. This is a necessary, but insufficient step - a co-receptor is also vital for proper cellular infection, and the virus uses a cytokine receptor on the surface of the host cell near the CD4 receptor to finish the lock-and-key entry process. It has been shown that individuals lacking the co-receptor have a natural immunity to infection by HIV [36], although it is not known how this receptor mutation arose. It has been observed that initial infection with HIV usually occurs in macrophages, as the predominately-transmitted strain of HIV is tropic for the cytokine coreceptor on macrophages [37, 38]. These important phagocytes and antigen presenting cells serve as a cloak for virus that can now be carried through the blood and lymph to all parts of the body (including crossing the bloodbrain barrier). As infection progresses, HIV expands its co-receptor usage repertoire, and can infect both $\mathrm{T}$ cells and macrophages. There is much evidence to support the observation that this infection process occurs more efficiently in activated cells, and that even when a resting cell binds virus, the viral DNA may never be inserted into the host genome [39, 40].

Once inside the host cell, the viral DNA can be inserted into the host-cell's genome only upon cellular activation, and new viral particles are created and released. For HIV, viral production per infected cell is estimated to be on the order of 500 virions per infected cell [41, 42]. It has been observed that these infected cells (whether resting or active) are at an increased risk for cell death via apoptosis or other pathways [43]. This enhanced cell death is also seen in bystander cells (cells not producing HIV) [44]. Infected cells are also cleared via a CTL response, or may die due to lysis from infection. 
As the infection develops, immune cells are depleted and a disruption in the immune system is established so that the host becomes susceptible to opportunistic infections and pathologies, like Pneumocystis carinii pneumonia (PNP), Kaposi's sarcoma, candidiasis, tuberculosis, etc. AIDS is diagnosed when the count in $\mathrm{CD}^{+} \mathrm{T}$ cells is less than $200 / \mathrm{mm}^{3}$ and/or the seropositive patient has one or more AIDS defining illnesses.

3.1. Modeling the mechanisms of HIV disease. Any theory or model of HIV disease progression needs to reconcile the following aspects of the disease process: (1) net loss of $\mathrm{CD} 4^{+}$lymphocytes is gradual and takes, on the average, 10 years; $(2) \mathrm{CD}^{+} \mathrm{T}$ cell numbers decrease in the blood earlier than in lymph nodes, and in the early symptomatic stages when $\mathrm{CD}^{+}{ }^{+}$lymphocytes are declining in the blood, their numbers are often increasing in the lymph nodes (lymphadenopathy); (3) there is some compartmentalization of HIV-infected cells to lymphatic tissues with the preponderance of cells producing virus residing there, as well as a large percentage of virus present on follicular dendritic cells; (4) the number of productively infected cells at any given time is very low, with approximately $10^{7}$ productively infected cells present in the whole body out of a total of $10^{12}$ lymphocytes (1:10 ${ }^{5}$ ratio); and (5) $\mathrm{CD} 4^{+}$lymphocytes that die, tend to die in lymph nodes, not in the peripheral blood, and those that are dying are mostly bystander cells. The factors determining the dynamics of the T-cell populations during HIV infection can be classified into 3 main areas: a change in cell productions, a change in cell migration patterns, and a change in cell life-spans (i.e. increased cell death). Each of these dynamics is modulated by cytokines as well as other influences. Two hallmarks of immunopathogenesis in the progression of HIV to AIDS are the loss of $\mathrm{CD} 4^{+} \mathrm{T}$ cell function in response to antigens and the critical reduction of $\mathrm{CD} 4^{+} \mathrm{T}$ cell numbers. It is probable that these two phenomena are related. Other data point to a reduction in $\mathrm{CD} 8^{+} \mathrm{T}$ cell anti-HIV activity associated with disease progression, and recent evidence indicates that CD8 $\mathrm{T}$ cells can be infected directly by HIV [45].

3.1.1. HIV-1 disease progression. As mentioned above, models of HIV-immune dynamics have gained much attention since the mid 1980s. The first three works done in this area were by Cooper [46], Dolezal and Hraba [47] and Perelson [48]. For recent reviews of HIV-immune models, see $[49,50]$. The Perelson et al. [25] model included populations of uninfected $\mathrm{CD}^{+} \mathrm{T}$ cells, latently infected $\mathrm{CD} 4^{+} \mathrm{T}$ cells, actively infected $\mathrm{CD}^{+} \mathrm{T}$ cells, and free HIV virus. This model provided a reasonable first approximation to the clinical progression of the disease, although the Tcell populations never declined to very low levels. (Note that this model is used in Section 4 of this tutorial.) This model was modified to include macrophages as a possible site of virus replication [21]; the result was a slower disease progression and a lower T-cell count, but the collapse of immunity (the "crash to AIDS") was always very abrupt. With both of these 
models chemotherapy was explored using a treatment that was assumed to reduce viral production.

To improve the mechanistic basis for HIV models, we included anatomical compartments other than the peripheral blood compartment in which viral loads and T-cell counts are typically measure. We first included the thymus as there is much evidence that the thymus is infected with HIV and that this may impair production of naive $\mathrm{CD} 4^{+} \mathrm{T}$ cells [51]. The compartment for the lymph system was included as there is evidence that lymph nodes become saturated early with infected T-cells and thus provides a constant supply of virus to the peripheral blood compartment throughout the infection $[42,52]$. Our results have given the most accurate simulations yet of the disease progression - including the slow decrease in $\mathrm{CD} 4^{+} \mathrm{T}$ cells, the quasi-constant viral load in peripheral blood until very late in the progression, and the long delay to the onset of AIDS [53].

3.1.2. Progression models of HIV. Any model of HIV disease progression should incorporate clinically determined dynamic information about the HIV-infected immune system. The essential elements are as follows. After an initial period of acute viremia in the first few weeks after seroconversion, $\mathrm{CD} 4^{+}$T-cell counts decline gradually from approximately 600 to $800 / \mathrm{mm}^{3}$ to almost $0 / \mathrm{mm}^{3}$ over approximately 10 years (normal $\mathrm{CD}^{+}$T-cell counts are 800 to $1200 / \mathrm{mm}^{3}$ ). Data from [54] is shown in Figure 1. The decline of $\mathrm{CD} 4^{+} \mathrm{T}$ cells is more rapid early in the infection [55]. Infected $\mathrm{CD} 4^{+} \mathrm{T}$ cells constitute $4 \%$ or less of the CD4 ${ }^{+} \mathrm{T}$-cell population [56]. The half-life of an infected $\mathrm{CD} 4^{+} \mathrm{T}$ cell is approximately 2 days $[5-7,57]$. After the initial viremia, plasma virus increases from below $10^{4} / \mathrm{ml}$ to $10^{7} / \mathrm{ml}$ or more during the variable course of infection with a sharper increase toward the end of the symptomatic phase [54]. The lifespan of a virus outside the cell is about $7.2 \mathrm{hrs}$ [5-7].

To model the longest stage of HIV infection, the asymptomatic phase, we developed a model $[19,18]$ consisting of ordinary differential equations for the variables $T(t)$ (the $\mathrm{CD}^{+}$T-cell population uninfected by virus at time $\mathrm{t}$ ), $T^{s}(t)$ (the $\mathrm{CD} 4^{+}$T-cell population infected by drug-sensitive virus (wild-type) at time $\mathrm{t}$ ), $T^{r}(t)$ (the $\mathrm{CD} 4^{+}$T-cell population infected by drug-resistant virus (mutant) at time t), $V_{s}(t)$ (the drug-sensitive virus population at time $\mathrm{t}$ ), and $V_{r}(t)$ (the drug-resistant virus population at time t). All these virus populations reside in the circulating blood, in which the values of uninfected $\mathrm{CD} 4^{+} \mathrm{T}$ cells and virus can be clinically measured. The equations are presented below (reprinted from [17]).

$$
\begin{aligned}
\frac{d T(t)}{d t} & =s(t)+p(t) T(t)-\mu_{T} T(t)-\mathbf{P}_{\mathbf{1}} k_{s} T(t) V_{s}(t)-k_{r} T(t) V_{r}(t) \\
\frac{d T^{s}(t)}{d t} & =k_{s} T(t) V_{s}(t)-\mu_{T_{i}^{s}} T^{s}(t)-p_{i}^{s}(t) T^{s}(t) \\
\frac{d T^{r}(t)}{d t} & =k_{r} T(t) V_{r}(t)-\mu_{T_{i}^{r}} T^{r}(t)-p_{i}^{r}(t) T^{r}(t)
\end{aligned}
$$




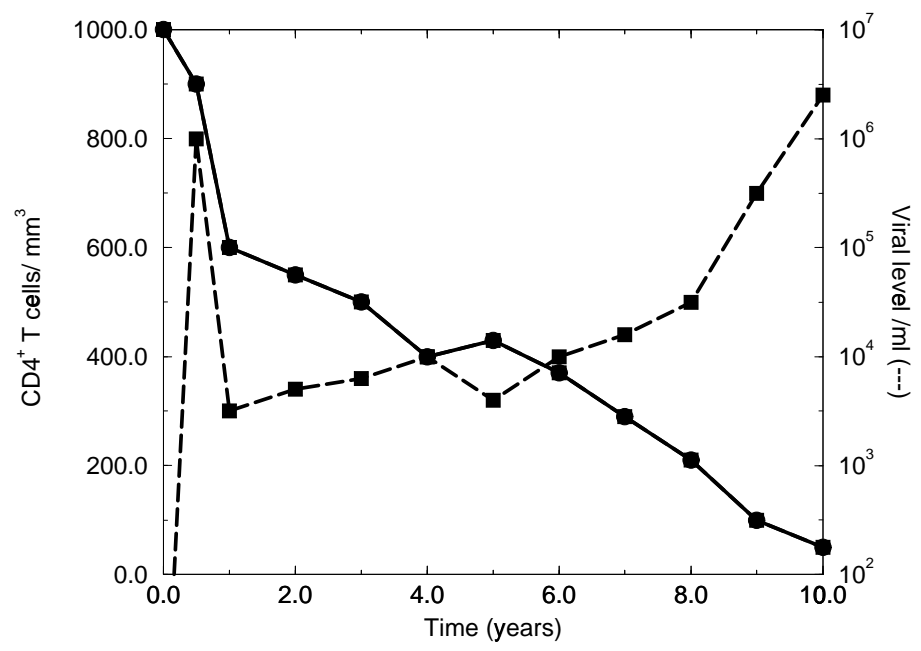

FIG. 1. Data graph taken directly from [54]. Shown are averaged $\mathrm{CD}_{4}^{+}{ }^{+}$-cell counts and viral loads from a large number of untreated patients.

(4) $\frac{d V_{s}(t)}{d t}=q \cdot p_{n}^{s}(t) T^{s}(t)+(1-q) \cdot p_{n}^{r}(t) T^{r}(t)-k_{T} T(t) V_{s}(t)+\mathbf{P}_{2} g_{s}(t)$,

(5) $\quad \frac{d V_{r}(t)}{d t}=q \cdot p_{n}^{r}(t) T^{r}(t)+(1-q) \cdot p_{n}^{s}(t) T^{s}(t)-k_{T} T(t) V_{r}(t)+g_{r}(t)$.

Initial conditions are $T(0)=T_{0}, \quad T^{s}(0)=0, \quad T^{r}(0)=0, \quad V_{s}(0)=$ $V_{s o}, \quad V_{r}(0)=0$. The model is explained as follows. The first two terms of Eqn. (1) represent the source of new T cells. This incorporates $\mathrm{T}$ cells from the bone marrow, thymus and general production. It is time dependent to represent the evidence that the source of new cells from the thymus, $s(t)$, is reduced during HIV infection [58]. The equation also includes a term to represent proliferative production (whether direct or indirect) due to the presence of antigen, $p(t) T(t)$. This production changes over the course of infection, which is accounted for in the time-dependent rate, $p(t)$. This general form allows for inclusion of different functional forms representing cell expansion. We choose to use Michaleis-Menten kinetics of the form $\frac{p V(t)}{V(t)+C}$. The other proliferation terms, $p_{i}^{j}(t)$ follow this same form. The next term is a natural death term, since cells have a finite lifespan; the average of which is $\frac{1}{\mu_{T}}$. The last two terms represent the infection of $\mathrm{CD}^{+} \mathrm{T}$ cells by both resistant and sensitive strains of virus. These terms are mass-action type terms with constant rates of infectivity $k_{s}, k_{r}$. We assume the law of mass action applies here based on the large numbers of cells and virus involved. $P_{1}$ represents the effects of treatment on viral infectivity in the plasma. Equations (2) and (3) describe changes in the infected population of CD4 $4^{+} \mathrm{T}$ cells, $T^{s}$ and $T^{r}$, respectively. (We assume 


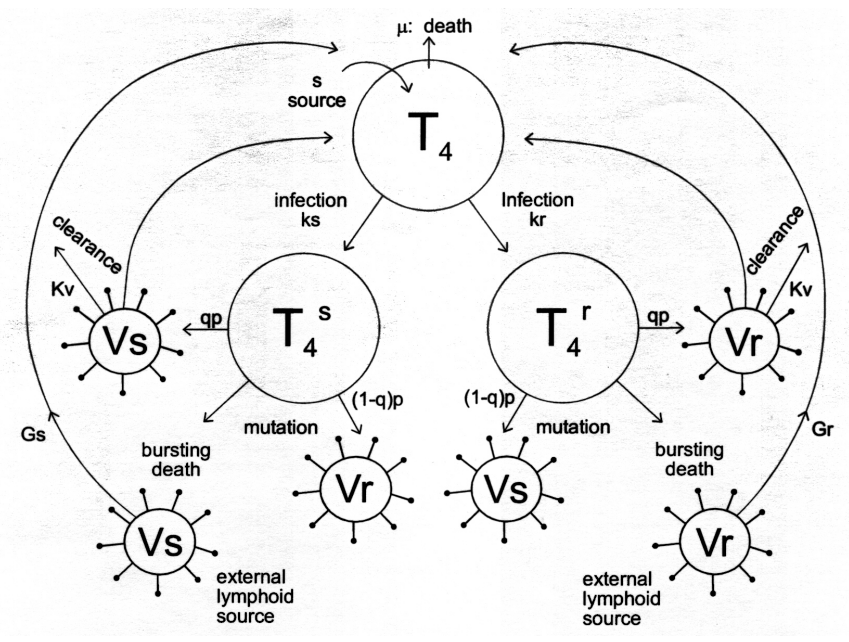

FIG. 2. Model of HIV-immune system dynamics. The uninfected $\mathrm{CD}_{4}^{+} \mathrm{T}_{\text {-cell }}$ population has a source rate of $s$, and a death rate of $\mu$. Cells are infected by either wild-type or resistant mutant virus, at rate $k$. These infected cells can produce either wild-type or mutant viral strains (which is dependent on the presence of drug for biased selection at rates $q$ and $p$ ). Both infected cells and virus are cleared by immune response and death. There is an external source of virus (both types) from the lymph system.

that, each $\mathrm{T}$ cell is dominated by, and hence producing, only one strain of the virus, either $V_{s}$ or $V_{r}$.) The first gain terms for each carries over from the loss terms in Eqn. (1). Then, infected cells are lost by such processes as natural death, viral killing, apoptosis or homing to LS.

In Equations (4) and (5), the first terms are the source for the virus population. Newly produced virion come from production by infected $\mathrm{CD} 4^{+} \mathrm{T}$ cells (from Eqs. (2) and (3)), in which new virion are produced at rates $p_{n}^{s}(t)$ and $p_{n}^{r}(t)$, respectively. We assume that upon each replication there is a probability of $(1-q)$ that a significant mutation will take place; and, that the proportion $q$ remain faithful to the original strain. We account for the mutation effects directly in the production term, although mutation occurs during the transcription stage which is not directly accounted for in the model.

The next two terms in Equations (4) and (5), $-k_{T} T(t) V(t)$ represent anti-HIV cytotoxic responses. As we track only CD4 ${ }^{+} \mathrm{T}$ cells in our model, and it is known that $\mathrm{CD} 8^{+} \mathrm{T}$ cells can directly kill infected cells, we wish to account for this cytotoxic response. Quantitative evidence supports that $\mathrm{CD} 8^{+} \mathrm{T}$ cells are present in numbers twice that of $\mathrm{CD} 4^{+} \mathrm{T}$ cells during HIV infection (the opposite ratio exists in uninfected individuals). Thus we use twice the number of $\mathrm{CD} 4^{+} \mathrm{T}$ cells to represent $\mathrm{CD} 8^{+} \mathrm{T}$ cells. We then use them in mass action form to represent direct interaction for killing to occur. 
As there is much evidence to support the major production of virus taking place in the lymphoid system (LS), we account for this phenomenon as a major contributor of virions, other than the small amount produced in the blood [52]. The input rates of lymphoid system virus are $g_{s}(t)$ and $g_{r}(t)$, for the sensitive and resistant viral strains, respectively. They are time dependent, as this will vary over the course of infection. We again use the Michaelis-Menten kinetics here as with the proliferation terms. $P_{2}$ represents the effects of treatment on viral infectivity in the LS. The model (1)-(5) describes the interaction of the immune system with HIV.

A typical untreated disease course (i.e. $P_{1}=P_{2}=1$ ) based upon $\mathrm{CD} 4^{+}$T-cell counts and viral load is simulated from the model in Figure 3. The initial virus level is determined by the model's parameters. This assumption is consistent with recent clinical findings that disease prognosis is correlated to a set-point of virus level established in each patient soon after the initial viremia, and viral levels and replication rates remain relatively stable after the set-point [59-62]. In the model, different set-points are obtained by varying key parameter values, such as viral burst size. This system is the only model to date that accurately captures clinic data [18]. This is now corroborated in published work from the Swiss HIV cohort study, where our model is used and gives consistent simulation of the clinical data during the long-term infection with HIV-1 [63].

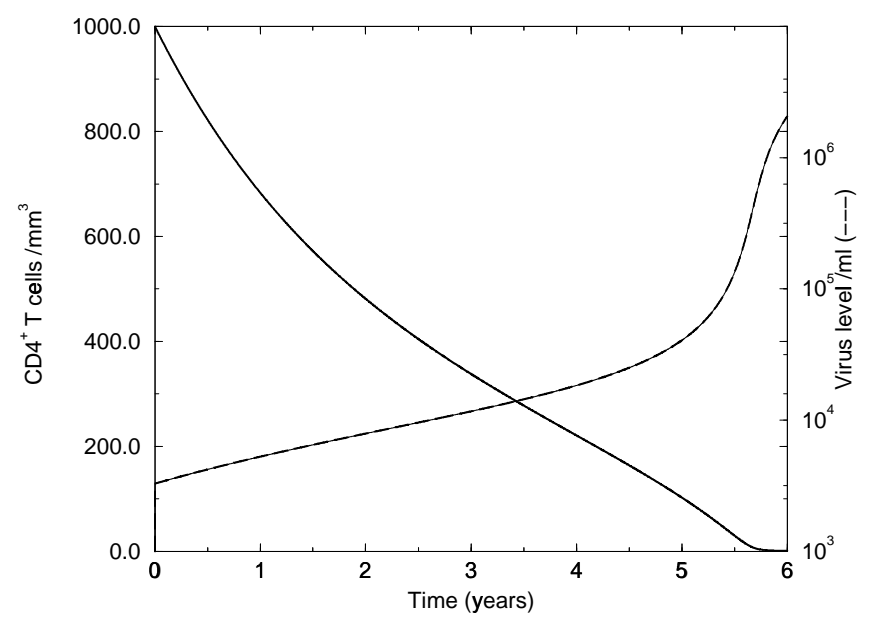

FIG. 3. A simulation of HIV dynamics for the model with initial T-cell count at $1000 / \mathrm{mm}^{3}$ and initial viral load at $10^{4} / \mathrm{ml}$. The curves correspond to data as shown in Figure 1. The set-point of the virus is in the middle range [61] and corresponds to a typical disease progression of about 6 years in this simulation.

3.2. Antiretroviral therapy of $\mathbf{H I V}$ infection. Clearly there is a necessity for both prevention and treatment of HIV infection. There are no 
vaccines as yet available, but there are several drugs now employed to lower viral loads and raise $\mathrm{CD} 4^{+} \mathrm{T}$-cell counts. These antiviral drugs fall into two main categories: inhibitors of reverse transcriptase and inhibitors of protease. The role of reverse transcriptase inhibitors is to interfere with the transcription of the RNA to DNA thus, halting cellular infection; the role of protease inhibitors is to interfere with post-translational viral particle assembly. Unfortunately, these drugs are not cures for the infection, but only serve as maintenance programs to temporarily prevent further progress of the virus. Despite this drawback, there is much clinical evidence to support the use of combinations of these chemotherapies in HIV infected individuals. Aside from the possibility of prolonging life in an HIV positive individual, it may make them less infectious to their sexual partners [64], as well as reduce rates of mother to fetus transmission [65]. Controversy exists among clinicians, however, as to whom should be treated, when they should be treated, what treatment scheme should be used, and if it fails, what should be done in replacement therapy.

HAART (highly active antiretroviral therapy) is a cocktail of different antiretroviral drugs. The effectiveness of HAART in terms of slowing progression to AIDS is high for a large percentage of cases although it presents several problems in terms of drug resistance and compliance.

Most chemotherapies reduce viral production in a dose dependent manner to the expense of multiple side effects as well as ineffectiveness of the treatment after a certain time due to the capability of the virus to mutate and become resistant [66].

3.2.1. Treatment models. Recent clinical trials have had some success in using combined drug therapy [67, 59]. Ongoing trials with combinations of drugs have shown sharp declines of viral counts to undetectable levels within several weeks of treatment; these levels are sustained for 1 year or more $[59,68,60]$. At the same time, $\mathrm{CD}^{+}$T-cell counts rise markedly before gradually leveling off $[59,68,60]$. This apparent remission of HIV infection offers hope for potential chronic control [57]. The longterm implications of patients on this therapy, however, are not yet fully understood. Mathematical models provide a means to understand the HIV infected immune system as a dynamic process. Models formulated as differential equations for the dynamic interactions of $\mathrm{CD} 4^{+}$lymphocytes and virus populations thus can be useful in identifying essential characteristics of HIV pathogenesis and chemotherapy.

3.2.2. Relevance of HIV-1 progression models to treatment and resistance. In the absence of any chemotherapy, it can be assumed that the sensitive strain (wildtype) of virus is dominant; but in the presence of treatment, selection is for resistant strains (mutant). Because of the development of resistance and side effects during single drug chemotherapy, the time frame for which the drug can be administered is finite $[69,70]$. This phenomenon can be modelled by treating, for example, for a 52 week 
window during the course of infection. Simulations of viral dynamics can be made using the total $\mathrm{CD} 4^{+} \mathrm{T}$-cell population over time together with HIV levels as indicators of drug effectiveness. To include monotherapy in the model, it is necessary to mathematically mimic the effects of a drug which is a reverse transcriptase inhibitor. Such drugs reduce viral infectivity, since they do not allow the viral DNA to be inserted into the host genome. Since multiple virions may infect a cell, such drugs may inhibit one virus, but allow another to get through or around the drug barrier. One can therefore model treatment by inhibiting the infectivity of the virus. This would affect both the viral infectivity of $\mathrm{T}$ cells, and also reduce the number of infectious virus flowing into the blood from the LS virus compartment. One can achieve this by multiplying the parameters $k_{s}$ and $g_{s}$ in the model by functions which are "off" outside the treatment period (thus having no effect) and "on" during the treatment period (thus reducing viral infectivity). Treatment functions which achieve this are of a piecewise continuous form with the value 1 outside the treatment period, and $P_{1}$ within the treatment period. $P_{1}$ is the treatment effectiveness, $0<P_{1}<1$. If $P_{1}=1$, treatment is $0 \%$ effective; if $P_{1}=0$, treatment is $100 \%$ effective. A similar function, with treatment parameter $P_{2}$, is also applied to the external LS, however with less effect (i.e. $P_{1}<P_{2}$, since it is proportionate reduction) as we assume the drug effectiveness is less there [52]. Drugs such as AZT reduce viral activity in a dose dependent manner. The efficacy of the chemotherapy may differ from patient to patient; therefore, $P_{i}$ for $i \in\{1,2\}$ represents the varying effectiveness of the drug in halting viral activity in a given patient.

Models of treatment by Kirschner and Webb (e.g. [19, 18]) have distinguished qualitatively two distinct outcomes as indicated by clinical trials. The first is resistance. Examples of resistance for three-drug combined therapy are reported for completed clinical trials by Collier et al. [71]. In these trials, there was on average an increase of CD4 ${ }^{+} \mathrm{T}$-cell counts by approximately $30 \%$ (peaking at approximately 8 weeks and returning to baseline at approximately 40 weeks) and a decrease of plasma virus by approximately $70 \%$ (peaking at approximately 4 weeks and recovering to half baseline at approximately 40 weeks). The second treatment outcome is remission. Examples of remission are indicated in preliminary reports of ongoing clinical trials $[59,60,39,72]$. In these trials, two things were observed: 1) plasma virus decreased sharply to undetectable levels in 2 to 4 weeks, and these levels are sustained for periods of 1 year or more, and 2) $\mathrm{CD} 4^{+}$T-cell counts increased steadily by $100 / \mathrm{mm}^{3}$ or more before gradually leveling off to below normal levels (this below normal recovery is believed to be due to an impaired production of new $\mathrm{CD} 4^{+} \mathrm{T}$ cells from the thymus and other sources [54,73]; this assumption is incorporated into the model).

Multi-drug treatment can be incorporated into the existing model as the reduction of two separate rates. The reduction of these rates provides 
treatment control variables corresponding to the intensity and velocity of HAART drug action. The variables are the rate at which virus infects uninfected $\mathrm{CD} 4^{+} \mathrm{T}$ cells and the rate of virus influx into the plasma from the lymph system. Reduction of this second rate is the most important for treatment outcome, since it is believed that as much as $98 \%$ of the virus in the circulating blood is contributed by the external lymphoid compartment $[59,60]$. In the simulations, the dynamics in the lymphoid compartment are modeled as a viral source term rather than mechanistically, since limited data are available for the host-pathogen dynamics in this compartment $[52,42]$. When treatment begins, the model assumes that a proportion of drug-sensitive virus mutates to drug-resistant virus. This proportion is also a treatment control variable corresponding to the combination of drugs used or the presence of genetic diversity at different disease stages [16]. Figure 4 shows the different outcomes of the treatment model simulations.
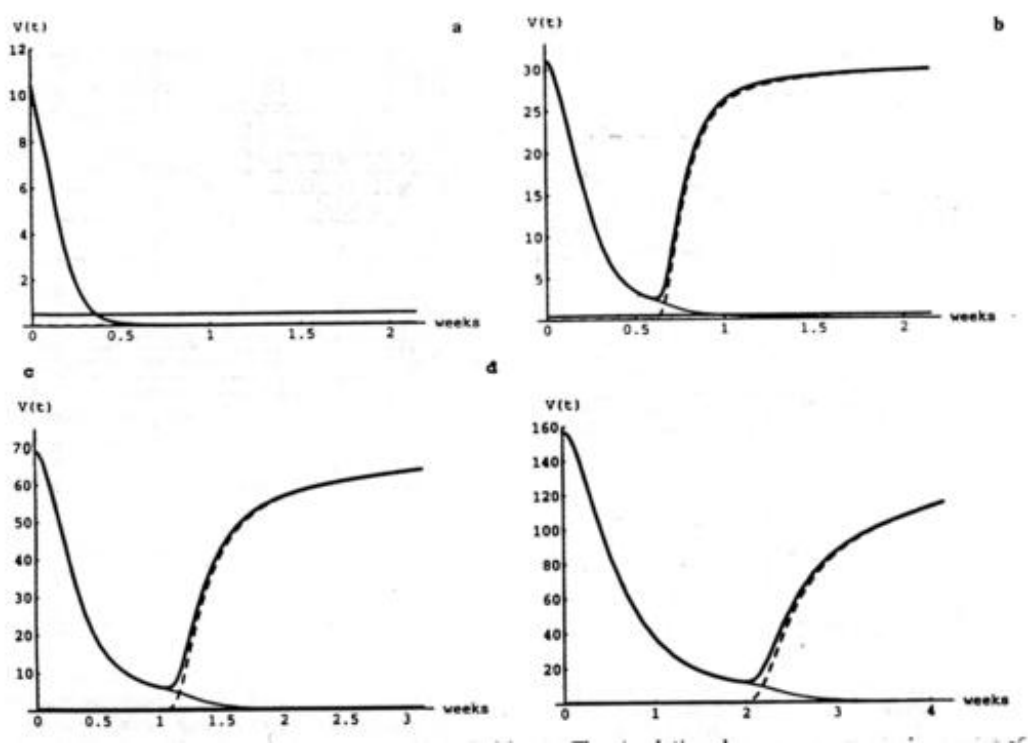

FIG. 4. Treatment simulations for four starting viral levels. The simulations have a common viral set-point of disease progression, and the treatment starting values are from Figure 3. Initiation of treatment with the lowest starting viral load achieves remission (a), while the other three develop resistance.

The model distinguishes primarily between resistance and remission in the assumption of a threshold condition for the virus population in the plasma (and thus for the virus population in the lymphoid compartment). The threshold condition is incorporated into the rate that controls the contribution of drug-resistant virus from the external lymphoid compartment to the plasma. When treatment drives the plasma virus level below the threshold, the drug-resistant virus population does not emerge, and the 
drug-sensitive virus population falls to undetectable levels. This threshold cannot be reached simply by gradually lowering the drug-sensitive virus population. Two additional factors must be considered: 1) when the virus population is above threshold, the high mutational capacity and short lifespan of the virus results in rapid production of drug-resistant variants; and 2 ) as the virus population approaches the threshold, Darwinian competition gives competitive advantage to the resistant viral strain as the sensitive viral strain diminishes in fitness and in numbers.

To reach the threshold, the virus population must be brought down extremely fast before mutation and selection pressure allow resistant virus to propagate in the drug-altered environment. In the simulations (see Figure 4, (reprinted from [17])), this rapid fall to the threshold can be achieved if treatment inhibits the rate of viral influx from the external lymphoid compartment term sufficiently fast. The threshold value depends on the drugs used and the capacity of the virus to mutate against these drugs. In some patients, plasma levels were reduced to undetectable levels, yet remission did not occur $[5,6]$. In these cases, there may have been an extremely low threshold specific to the drugs used or a disproportionately lower suppression of virus in the lymphoid compartment than in the plasma.

Although computer models of HIV therapy are no substitute for clinical trials, they can bring into focus essential elements of the dynamic processes involved. The treatment simulations presented here identify the following qualitative dynamic elements involved in resistance and remission: 1) remission can occur if the viral production in the lymphoid tissues is suppressed below a threshold level; 2) drug action must be strong enough and fast enough to drive the virus population to the threshold before resistant virus appears and propagates; 3 ) combination therapy or early treatment lowers the capacity of the virus to mutate to resistant strains and thus forestalls their emergence until the threshold is reached. In the next section we present a different approach to modeling of chemotherapy in HIV.

4. A chemotherapy feedback control model. The question of whether or not treatment should be given in a constant or dynamic way remains open. Some scientists believe that it is too difficult to taylor treatment regimes for individual patients. Others believe that in life-threatening diseases such as HIV, we should treat each patient with all available options. To this end, Kirschner et al. [66] used optimal control methods to explore the issue of a dynamic treatment regime. They addressed this problem concentrating on when and how treatment should be initiated for patients who are in the early stages of infection where uninfected cell counts are high. Their optimal control approach has an objective function based on benefit, measured as the number of uninfected cell counts less the systemic cost of chemotherapy. Using a previously studied simple model of HIV-T cell dynamics ([25] given in (6-9) below), the authors concluded that a dynamically scheduled treatment protocol can maintain the uninfected cell counts in normal levels while reducing the side effects of chemotherapy. 
Optimal control based solutions lack robustness against modeling errors and imperfect measurements, and depend strongly on the model used to compute the treatment scheduling. Host HIV dynamics are poorly understood. Therefore, misconstructed model dynamics and uncertainty in parameters may lead to faulty conclusions, such as failure of treatment due to unnecessary high doses or high virus counts due to low doses. We now present a different approach to HIV chemotherapy modeling using robust control summarizing the results from [74].

An alternative to optimal control strategies is the method of feedback control. The aim of this section is to summarize recent results that explore the use of robust control theory to design treatment scheduling for the treatment of HIV infected patients. Alvarez-Ramirez et al. [74] considers the percentage effect of the chemotherapy on viral production as the target variable seeking to regulate the viral production via a feedback function where the percent of effect of chemotherapy is computed from actual measurements of viral and cells counts.

4.1. The HIV-immune system model. To illustrate the possibilities of the application of feedback control, the authors in [74] make use of an early model for HIV dynamics from Perelson et al. [25] (given in (6-9) below), which was also used for the optimal control strategies applied to treatment as described above [66].

In the model, $T$ denotes the numbers of uninfected CD4 ${ }^{+} T$ cells per $\mathrm{mm}^{3}, T_{1}$ and $T_{2}$ denote respectively the numbers of both latently infected (cells that contains virus but do not release them) and actively infected $\mathrm{CD}^{+} T$ cells (virus releasing cells) per $\mathrm{mm}^{3}$. The number of free infectious virus particles per $\mathrm{ml}$ is $V$. Definitions and numerical information for the parameters can be found in Table 2 of Kirschner et al. [66].

$$
\begin{aligned}
\frac{d T(t)}{d t} & =\frac{s}{1+V(t)}-\mu_{T} T(t)+r T(t)(1-f(t))-k_{1} T(t) V(t), \\
\frac{d T_{1}(t)}{d t} & =k_{1} T(t) V(t)-\mu_{T} T_{1}(t)-k_{2} T_{1}(t) \\
\frac{d T_{2}(t)}{d t} & =k_{2} T_{1}(t)-\mu_{b} T_{2}(t) \\
\frac{d V(t)}{d t} & =N \mu_{b} T_{2}(t)-k_{1} V(t) T(t)-\mu_{V} V(t),
\end{aligned}
$$

where $f(t)=\left(T(t)+T_{1}(t)+T_{2}(t)\right) / T_{\max }$.

The model is briefly explained as follows (for a more complete description see [25]). The first term of Equation (6) represents the source of new $\mathrm{CD} 4^{+} T$ cells from the thymus. As in the first model (see Eqn. 1) this term is a function of viral load to represent the reduction in the number of newly produced $\mathrm{T}$ cells during HIV infection [58]. The next term is a natural death term, followed by a term representing the stimulation of $\mathrm{CD}^{+} T$ to proliferate in the presence of virus. The last term represents 
the infection of $\mathrm{CD} 4^{+} T$ cells by virus and is determined by the rate of encounters of $\mathrm{CD}^{+} T$ cells with virus. This is based on the law of mass action. In Equation (7), latently infected CD4 ${ }^{+} T$ cells are assumed to have the same death rate $\mu_{T}$ as uninfected $\mathrm{T}$ cells, although other factors can augment their death rate [66]. Equation (8) models the rate of change of actively infected $\mathrm{CD} 4^{+} T$ cells. The first term represents the rate at which latently infected cells become actively infected cells. Actively infected cells produce virus and die at per capita rate $\mu_{b}$. Equation (9) models the rate of change of free virus population. The model assumes that when an actively $\mathrm{CD} 4^{+} T$ cell becomes activated through exposure to antigen, replication of the virus is initiated and $N$ virus are produced before the host cell dies. The middle term in the viral equation represents the idea that, as we are tracking only free viral particles in $V(t)$, The term $-\mu_{V} V$ accounts for viral loss and/or removal from the body. In the absence of virus, the $T$ cell population has a unique steady state known as the uninfected steady state such that $T_{0}>0$. Reasonable initial conditions for the differential equations system are $T(0)=T_{0}, T_{1}(0)=T_{1}^{0}, T_{2}(0)=T_{2}^{0}$ and $V(0)=V_{0}$. In particular, if the initial innoculum is free virus and not infected cells, then $T_{1}(0)=T_{2}(0)=0$.

The system (6)-(9) has a second steady state [25]. The uninfected steady state represents a healthy individual, while the second steady state corresponds to a successful invasion of HIV (infected steady state), in which uninfected $\mathrm{T}$ cells have a low, positive concentration. It has been shown in [25] that if the parameter $N$ is below a critical value $N_{c}$, the uninfected steady state is stable and the infected steady state is unstable. If $N<N_{c}$, the uninfected steady state is globally asymptotically stable. At $N=N_{c}$ a transcritical bifurcation occurs. Once $N>N_{c}$ stability is reversed, and the infected state is locally stable. Model (6)-(9) can be used to study the effects of chemotherapy $[66,25]$ by representing the effect of drug treatment as a reduction of the viral production rate as shown in [66]. Obviously, if chemotherapy reduces the number of virus production below the value $N$, then the immune system could recover and lead to the uninfected steady state. Thus, a treatment scheduling design is required to avoid proliferation of free infectious virus particles.

4.2. The feedback treatment model. The control variable represents the percentage of effect the chemotherapy has on viral production. The authors in $[74,66,25]$ mimic the effects of HAART, by assuming that chemotherapy reduces viral load; thus in Equation (9), the control $u(t)$ multiplies the number of free virus produced by $T_{2}(t)$ cells:

$$
\frac{d V(t)}{d t}=u(t) N \mu_{b} T_{2}(t)-k_{1} V(t) T(t)-\mu_{V} V(t) .
$$

We require the control to satisfy the restriction $0 \leq u(t) \leq 1$ where $u(t)=1$ is the maximal use of chemotherapy. For design purposes, it is 
assumed that $N>N_{c}$, so the model does not evolve in the region of stability of the uninfected state (i.e. the individual has active HIV infection). Kirschner et al. [66] defined a control objective functional that maximizes chemotherapy benefits based on the uninfected T-cell counts. AlvarezRamirez et al [74] use a different control objective: the regulation of the free infectious virus particles about a low reference value by scheduling the chemotherapy dosage. This follows since a reduction of free infectious virus leads to a reduction of (latently and active) infected cells and, consequently, to an increase in uninfected cells. A feature of this approach is that the virus reference value is a design variable that can be prescribed in terms of the degree of infection and patient characteristics. As pointed out in [66], public health policies are being designed to determine the time and degree of infection of each particular case and therefore health officials may want to treat under asymptomatic or symptomatic infection scenarios depending on viral load.

If $V_{r}$ denotes the virus reference value, then it is assumed that the dynamic behavior of the free infectious virus particles is:

$$
\frac{d V}{d t}=-\tau_{c}^{-1}\left(V(t)-V_{r}\right),
$$

where $\tau_{c}>0$ is a closed loop time constant. Notice that the dynamics of the above equation are asymptotically stable around the reference value $V_{r}$ with $\tau_{c}$ as the convergence time. The feedback control law is based on a matching scheme. Provided $N \mu_{b} T_{2} \neq 0$ for all positive $t$, exact model matching between (10) and (11) is achieved by the following feedback function:

$$
u(t)=\left(k_{1} V(t) T(t)+\mu_{V} V(t)-\frac{d V(t)}{d t}\right) / N \mu_{b} T_{2}(t) .
$$

The above function corresponds to feedback treatment since it is a function of the actual values of actively infected cell concentration $T_{2}(t)$ and of free infectious virus particles $V(t)$. Under the above control (12), the controlled dynamics are given by system (6-8) plus Equation (11). It can be proved that feedback control $u(t)$ given above can regulate the immune system-HIV dynamics to either steady state.

A major drawback of the feedback control function (12) is that perfect knowledge of the parameters and dynamics are required for its implementation. It is in this sense that the feedback function (12) is called an ideal scheduling treatment. The model above is only an approximation of the real immune system-HIV dynamics and in fact, it does not account for some key aspects of the dynamics. In particular, parameter estimation from actual clinical data parameters is inexact as well as time varying, and even some model parameters are unobtainable from clinical data. For instance, only rough estimates of the number of free virus produced by $T_{2}(t)$ cells are presently available. As in the case of optimal control based approaches [66], the design of treatment scheduling under these uncertainties may lead 
to undesired results (e.g. premature ineffectiveness of treatment due to unnecessary high doses or high virus counts due to low doses). The aim of this section is to use control theory tools to design a robust treatment scheduling in the face of model uncertainties.

The following assumptions are made for control design purposes:

- Free infectious virus particles and actively infected cell concentrations are available from (periodic) measurements. This is a reasonable assumption, since cell and virus counts can be obtained with present clinical techniques.

- Estimates of $N, \mu_{b}, \mu_{V}$ are known denoted by $\hat{N}, \hat{\mu}_{b}, \hat{\mu}_{V}$ respectively. Clinical data are becoming more accessible, making it possible to get estimates for them.

We write the viral dynamics, $V(t)$, as

$$
V^{\prime}(t)=-\mu_{V} V(t)+\eta\left(V(t), T_{2}(t), u(t)\right)+\hat{\beta} u(t),
$$

where $\hat{\beta}=\hat{N} \hat{\mu}_{b}$ and

$\eta\left(V(t), T_{2}(t), u(t)\right)=-\left(\mu_{V}-\hat{\mu}_{V}\right) V(t)-k_{1} V(t) T(t)+\left(N \mu_{b}-\hat{\beta}\right) T_{2}(t) u(t)$

is the model error function. The aim is to estimate $\eta\left(V(t), T_{2}(t), u(t)\right)$ and to design a feedback function to approximate the optimal scheduling treatment function (12).

To estimate the model error $\eta(t)$, we introduce the observer

$$
\hat{\eta}^{\prime}(t)=\omega_{e}(\eta(t)-\hat{\eta}(t))
$$

where $\hat{\eta}(t)$ is the estimate of $\eta(t)$ and $\omega_{e}>0$ is the estimation frequency, which is an adjustable parameter. If $e=\eta-\hat{\eta}$ is the estimation error then $e^{\prime}=-\omega_{e} e+\eta^{\prime}$. If the model error dynamics are bounded the idea is to pick a sufficiently large estimation frequency value and push the estimate modeling error towards the real modeling error. The implementation of this idea can be consulted in [74]. We now proceed to some numerical examples.

4.2.1. Numerical simulations. In this section we study the controlled immune system HIV dynamics numerically to illustrate our treatment scheduling design as well as to provide evidence that our feedback control design is likely to work in more general situations. We assumed the following scenarios:

- Free virus and actively infected cell concentrations are measured every $P$ days. Moreover, such measurement is delayed $P$ days. This means that the sample for measurement is taken every $P$ days and the actual measurement is available at the next sampling instant.

- An upperbound for the parameter $N$ is $\hat{N}=1800$ virus counts/cell [25]. Note that the estimated value $\hat{N}=1800$ virus counts/cell has about $80 \%$ error with respect to the real value. 
- We use the estimate $\hat{\mu}_{b}=0.3 / d$, such that the death rate of the $T_{2}(t)$ cell population is overestimated about $25 \%$.

- Chemotherapy begins $D$ days after the onset of infection. The estimated parameter becomes $\hat{\beta}=240$ virion counts cell ${ }^{-1} \mathrm{~d}^{-1}$.

Initial conditions are: $T(0)=1000$ cells mm $^{-3}, T_{1}(0)=T_{2}(0)=0$ cell $\mathrm{mm}^{-3}$, and $V(0)=0.001$ particles $\mathrm{mm}^{-3}$. The control parameters are: $\tau_{c}=7$ and $\omega_{e}=0.05 \mathrm{~d}^{-1}$; therefore, the desired reference value can ideally be achieved in about a week, and the cutoff period of the modeling error estimation about two weeks. Thus, system parameter changes can be detected in at most two weeks.

For comparison purposes the dynamical behavior of the uninfected cells and virus populations in absence of treatment are presented in Figure 5. Notice that for this set of parameters, the stage where the uninfected cell concentration decreases rapidly starts $2-2.5$ years after the onset of infection. In the first year, the virus and infected cell concentration are very small. This can be a drawback for treatment scheduling based on feedback control because the modeling error estimator may be insensitive to excessively small values of virus concentrations. Based on the numerical simulation displayed in Figure 5, we propose to use our feedback control scheme for treatment scheduling about 400 days after the onset of infection.
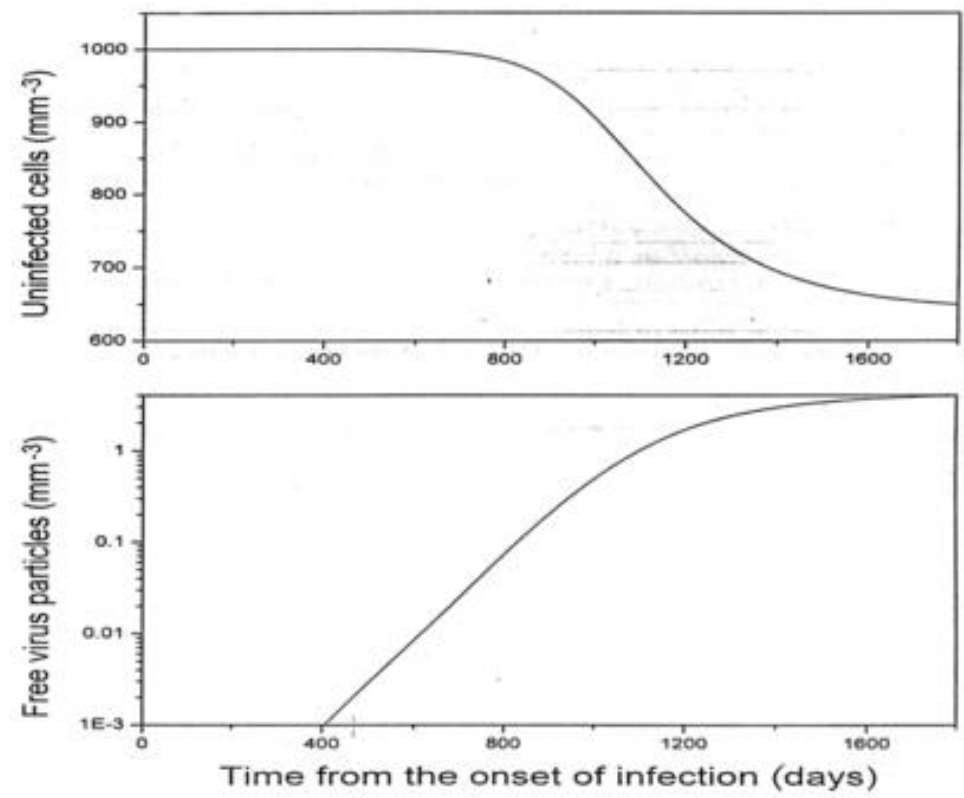

FIG. 5. Time series plot of the solution to the model in Equations (6-9) showing the virus and uninfected T-cell populations. 
Let the reference $V_{r}=0.05$ particles $\mathrm{mm}^{-3}$, correspond to a steady state with about a $10 \%$ decrease in the normal uninfected T-cell population. Figure 6 presents the dynamics of the immune system-HIV interactions under chemotherapy computed from the proposed feedback control for three different values of the sampling period $P$; namely, 7,15 and 30 days, and $D=1000$, corresponding to the beginning of the catastrophic stage of fast, uninfected $\mathrm{T}$ cell decrease. Examining the results, two things are evident. First, the reference value $V_{r}$ is achieved in about 250 days. After this time, the treatment achieves a stationary value. Second, the smaller the sampling period, the better the performance of the chemotherapy administration. This point highlights the importance of monitoring frequently the evolution of $T$ cell populations in HIV infected patients.
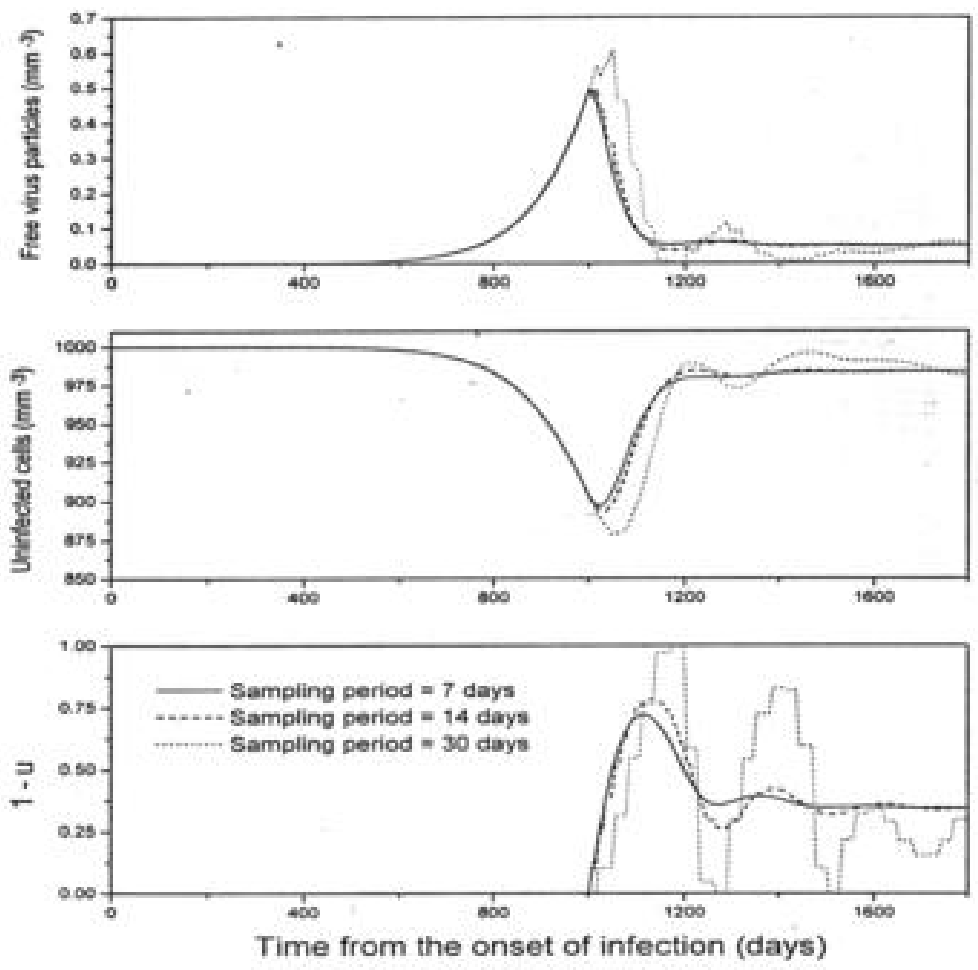

FIG. 6. Dynamics of the immune-HIV interaction for different reference values of the sampling period, $P$ at 7, 15 and 30 days.

We also did studies where we varied the treatment initiation times, $D$ at times 1500,1000 and 2000 days. These results reveal that early treatment leads to moderate treatment scheduling. On the other hand, 
late treatment induces the highest dose possible for long periods, which may lead to premature resistance (data not shown).

Finally, we studied the performance of the feedback-control based treatment under the instantaneous development of viral resistance to chemotherapy (data not shown). To this end, we assumed a successive $15 \%$ sudden change at day 1500 of the parameter $\hat{N}$; the number of free virus produced by $T_{2}(t)$ cells. Although the parameter $\hat{N}$ can not verified from clinical data, the modeling error estimator is able to detect these changes. In this case after a sudden change in $\hat{N}$, the control feedback has to rectify the steady state value of chemotherapy, which increases as $N$ also increases. These results suggest that the steady state value of the control, say $u$, can be used to monitor mutations and resistance of the virus to drugs. In this way, large values of $u$ may indicate adaptation of the virus to chemotherapies.

It should be stressed that the numerical results presented above do not imply that the feedback control approach to HIV chemotherapy proposed in this work is a finished methodology. Instead, our objective has shown the strength of using control theory tools within a cross disciplinary framework to attack problems in medicine.

Acknowledgements. JXVH acknowledges support from a CONACYT (1998) grant and finished his part of this work while on sabbatical leave at the Seccion de Metodologia y Teoria de la Ciencia CINVESTAVIPN. DEK acknowledges support from NIH grant \# R01 HL62119.

\section{REFERENCES}

[1] R. Medzhitov and C.A. JR. Janeway. Innate immunity: impact on the adaptive immune response. Curr. Opin. Immunol, 9:4-9, 1997.

[2] F.M. BuRnet. The clonal selection theory of acquired immunity. Cambridge University Press, UK, 1959.

[3] P. Matzinger. Tolerance, danger and the extended family. Ann. Rev. Immunol, 12:991-1045, 1994.

[4] J.P. RIDGE, E.J. FUChS, AND P. MATZINGER. Neonatal tolerance revisited: turning on newborn T cells with dendritic cells. Science, 271:1723-1726, 1996.

[5] D.D. Ho, A.U. Neumann, A.S. Perelson, And et AL. Rapid turnover of plasma virions and CD4 lymphocytes in HIV-1 infection. Nature, 373:123-126, 1995.

[6] X. Wei, S.K. Ghosh, M.E. TAYlor, V.A. Johnson, AND et AL. Viral dynamics in HIV-1 infection. Nature, 373:117-122, 1995.

[7] A.S. Perelson, A.U. Neumann, M. Markowitz, and et al. HIV-1 dynamics in vivo: Virion clearance rate infected cell life span and viral generation time. Science, 271:1582-1586, 1996.

[8] R.W. Anderson, M.S. Ascher, ANd H.W. Sheppard. Direct HIV cytopathicity cannot account for CD4 decline in AIDS in the presence of homeostasis: A worst-case dynamical analysis. J. AIDS and Human Retrov., 17:245-252, 1998.

[9] R.W. ANDERSon. How adaptive antibodies facilitate the evolution of natural antibodies. Immun. Cell Biol., 74:286-291, 1996.

[10] Z AGUR. A new method for reducing cytotoxicity of the anti-AIDS drug AZT. Biomedical Modeling and Simulation ed. D.S. Levine. J.C. Baltzer A.G. Scientific Publishing Co. IMACS, 1:59-61, 1989. 
[11] M.A. NOWAK, R.M. MAY, AND R.M. ANDERSON. The evolutionary dynamics of HIV-1 quasispecies and the development of immunodeficiency disease. AIDS, 4:1095-1103, 1990.

[12] M.A. NowaK AND R.M. MaY. Mathematical biology of HIV infections: Antigenic variation and diversity threshold. Math. Biosciences, 106:1-21, 1991.

[13] M.A. NowaK AND R.M. MaY. Coexistence and competition in HIV infection. $J$. Theoret. Biol., 159:329-342, 1992.

[14] M.A. Nowak And C.R.M. Bangham. Population dynamics of immune responses to persistent viruses. Science, 272:74-79, 1996.

[15] A. MCLEAN AND M. NOWAK. Interactions between HIV and other pathogens. $J$. Theoret. Biol., 155:69-86, 1991.

[16] A. McLean ANd M. NowaK. Competition between AZT sensitive and AZT resistant strains of HIV. AIDS, 6:71-79, 1992.

[17] D Kirschner ANd G.F. WebB. A model for treatment strategy in the chemotherapy of AIDS. Bulletin of Mathematical Biology, 58(2):367-390, 1996.

[18] D KirSChNeR AND G.F. WebB. Qualitative differences in HIV chemotherapy between resistance and remission outcomes. Emerging Infectious Diseases, $3(3): 273-283,1997$.

[19] D. KiRSChNeR AND G.F. WeBB. Understanding drug resistance in the monotherapy treatment of HIV infection. Bulletin of Mathematical Biology, 59(4):763-785, 1997.

[20] D KirSChNer AND G.F. WebB. A mathematical model of combined drug therapy of HIV infection. Journal of Theoretical Medicine, 1, 1997.

[21] D KIRSChNer AND A PeRELSON. A model for the immune system response to HIV: AZT treatment studies. Mathematical Population Dynamics, 1:296-310, 1995.

[22] R.M. ANDERSON AND R.M. May. Complex dynamical behaviour in the interaction between HIV and the immune system. Cell-to-cell signalling: experiments to theoretical models, pages 335-349, 1989.

[23] N. Stilianakis, K. Dietz, And D. Schenzle. Analysis of a model for the pathogenesis of aids. Math. Biosci., 145:27-46, 1997.

[24] R.J. DeBoer and A Perelson. Target cell limited and immune control models of HIV infection: A comparison. J. theoret. Biol., 190:201-214, 1998.

[25] A. Perelson, D. Kirschner, and R. De Boer. The dynamics of HIV infection of CD4+ T-cells. Journal of Mathematical Biosciences, 114:81-125, 1993.

[26] A. Perelson and P. Nelson. Mathematical analysis of HIV-1 dynamics in vivo. SIA M Review, 41:45-76, 1999.

[27] D. Kirschner AND G.F. WebB. Immunotherapy of HIV-1 infection. J. Biol. Systems, 6:71-83, 1997.

[28] A.U. Neumann, N.P. Lam, H. Dahari, M. Davidian, T.E. Wiley, B.P. Mika, A.S. Perelson, and T.J. LAYden. Differences in viral dynamics between genotypes 1 and 2 of hepatitis C virus. Journal of Infectious Diseases, 182:2835,2000 .

[29] A.U. Neumann, N.P. Lam, H. Dahari, D.R. Gretch, T.E. Wiley, T.J. Layden, AND A.S. Perelson. Hepatitis $\mathrm{C}$ viral dynamics in vivo and the antiviral efficacy of IFN- $\alpha$ therapy. Science, 282:103-107, 1998.

[30] S.J. Merrill. AIDS: Background and the dynamics of the decline of immunocompetence. Theoretical Immunology, Part Two: A.S. Perelson ed., pages 59-75, 1987.

[31] S.J. MerRILL. Modeling the interaction of HIV with cells of the immune response. Mathematical and Statistical Approaches to AIDS Epidemiology: C. CastilloChavez ed., 83:371-385, 1989.

[32] W.Y. TAN. Estimation of HIV infection and incubation in space state models. Math. Biosci., 167:31-50, 2000.

[33] W.Y. TAN. Some state space models of HIV pathogens under treatment by antiviral drugs in HIV infected individuals. Math. Biosci., 156:69-94, 1999. 
[34] W.Y. TAN. Stochastic models of the dynamics of cd4 + T cells infected by HIV and some monte carlo studies. Math. Biosci., 147:173-205, 1998.

[35] F. BARR-SinOuSSI AND ET AL. Isolation of a T-cell lymphotropic virus from a patient at risk for the acquired immunodeficiency syndrome (AIDS). Science, $220: 868-871,1983$.

[36] M.P. D'SouzA AND V.A. HARdEN. Chemokines and HIV-1 second receptors: Confluence of two fields generates optimism in AIDS research. Nature Medicine, $2: 1293-1300,1996$.

[37] M.T.L. Roos, J.M.A. LANGe, R.E.Y. DEGoede, AND ET AL. Viral phenotype and immune response in primary HIV-1 infection. J Infect Dis, 165:427-432, 1992.

[38] T. Zhu, H. Mo, N. Wang, D.S. Nam, Y. CaO, AND et AL. Genotypic and phenotypic characterizations of HIV-1 in patients with primary infection. Science, 261:1179-1181, 1993.

[39] G. Pantaleo, C. Graziosi, J.F. Demarest, L. Butini, M. Montroni, C.H. Fox, AND ET AL. HIV infection is active and progressive in lymphoid during the clinically latent stage of disease. Nature, 362:355-358, 1996.

[40] T.W. Chun, L. Carruth, D. Finzi, X. Shen, and et al. Quantification of latent reservoirs and total body viral load in HIV-1 infection. Nature, 387:183-187, 1997.

[41] W. Cavert, D.W. Notermans, K. Staskus, and et al. Kinetics of response in lymphoid tissues to antiretroviral therapy of HIV-1 infection. Science, 276:960 964,1997

[42] A.T. HaAse, K. Henry, M. Zupancic, And et AL. Quantitative image analysis of HIV-1 infection in lymphoid tissue. Science, 274:985-989, 1996.

[43] J. Estaquier, . Tanaka, T. Suda, S. Nagata, P. Golstein, and J.C. Ameisen. Fas-mediated apoptosis of CD4+ and CD8 + T cells from human immunodeficiency virus-infected persons: differential in vitro preventive effect of cytokines and protease antagonists. Blood, 87:4959-4966, 1996.

[44] M. Clerici, J.A. Sarin, A. Sanderzofsky, A.L. Landay, H.A. Kessler, F. Hashemi, C.W. Hendrix, S.P. Blatt, J. Rusnak, M.J. Dolan, R.L. Coffman, P.A. Henkart, AND G.M. Shearer. Antigen-stimulated apoptotic T-cell death in HIV infection is selective for CD4+ T cells, modulated by cytokines and effected by lymphotoxin. AIDS, 10:603-611, 1996.

[45] K. SAha, K.J. Zhang, A. Gupta, R. Dave, M. Yimen, and B. Zerhouni. Isolation of primary HIV- 1 isoltates that target $\mathrm{CD}^{+} \mathrm{T}$ cells using CD8 as a receptor. Nature Medicine, 7:65-73, 2001.

[46] L.N. CoOper. Theory of an immune system retovirus. Proc. Natl. Acad. Sci., 83:9159-9163, 1986.

[47] J. Dolezal and T. Hraba. Application of mathematical model of immunological tolerance to HIV infection. Folia Biologica, 34:336-340, 1988.

[48] A.S. Perelson. Modeling the interaction of the immune system with HIV. Mathematical and Statistical Approaches to AIDS Epidemiology: Lecture Notes in Biomathematics, 83:350-370, 1989.

[49] A.S. Perelson And P.W. Nelson. Matematical analysis of HIV-1 dynamics in vivo. SIAM Review, 41:3-44, 1998.

[50] D. COVERT AND D. Kirschner. Revisiting the early models of the host-pathogen interactions in HIV infection. Comments on Theoretical Biology, 5:383-411, 2000 .

[51] D. Kirschner, R. Mehr, And A. Perelson. The role of the thymus in pediatric HIV-1 infection. J. AIDS and Hum. Retrov., 18:95-108, 1998.

[52] A. Lafeuillade, C. Poggi, N. Profizi, and et Al. Human immunodeficiency virus type 1 in lymph nodes compared with plasma. J. Infec. Diseases, 174:404-407, 1996.

[53] D. Kirschner, G.F. WebB, AND M. Cloyd. A model of HIV-1 disease progression based on virus-induced homing and and homing induced apoptosis of $\operatorname{cd} 4^{+} \mathrm{T}$ cells. J. AIDS and Human Retro., 24:352-362, 2000. 
[54] E. Pennisi And J. Cohen. Eradicating HIV from a patient: not just a dream? Science, 272:1884, 1996.

[55] A.N. Philips, C.A. Sabin, A. Mocroft, and G. Janossy. Antiviral therapy. Nature, 375:195, 1995.

[56] J. Embretson, M. Zupancic, J.L. Ribas, R.A. Burke, P. Racz, K. TennerRACZ, AND A.T. HAASE. Massive covert infection of helper T lymphocytes and macrophages by HIV during the incubation period of AIDS. Nature, 362:359-362, 1993.

[57] J.M. CofFIN. HIV population dynamics in vivo: implications for genetic variation, pathogenesis and therapy. Science, 267:483-489, 1995.

[58] D.C. Douek, R.D. McFarland, P.H. Keiser, And et Al. Changes in thymic function with age and during the treatment of HIV infection -see comments. Nature, 396:690-695, 1998.

[59] D.D. Richman. HIV therapeutics. Science, 272:1886-1887, 1996.

[60] C.J. Carpenter, M.A. Fischl, S.M. Hammer, M.S. Hirsch, D.M. Jacobsen, D.A. Katzenstein, AND ET AL. Antiretroviral therapy for HIV infection. JAMA, 276:146-154, 1996.

[61] D. Ho. Viral counts in HIV infection. Science, 272:1124-1125, 1996.

[62] J.W. Mellors, C.R. Rinaldo, P. Gupto, R.M. White, J.A. Todd, And L.A. KINGSELY. Prognosis in HIV-1 infection predicted by the quantity of virus in plasma. Science, 272:1167-1170, 1996.

[63] R.W. Cone, P. Gowland, M. Opravil, P. Grob, B. Ledergerber, and et Al. Levels of HIV-infected peripheral blood cells remain stable throughout the natural history of HIV infection. AIDS, 12:2253-2260, 1998.

[64] D.J. Anderson, T.R. O'Brien, J.A. Politch, A. Martinez, G.R. Seage, and ET AL. Effects of disease stage and azt therapy on the detection of HIV-1 in semen. JAMA, 267:2769-2774, 1992.

[65] M. Nozyce, M. Hobberman, S. Arpadi, A. Wiznia, G. Lambert, and et al. A 12-month study of the effects of oral AZT on neurodevolopmental functioning in a cohort of vertically HIV-infected inner-city children. AIDS, 8:635-639, 1994.

[66] D. Kirschner, S. Lenhart, ANd S. Serbin. Optimizing chemotherapy of HIV infection: Scheduling, amounts and initiation of treatment. Journal of Mathematical Biology, 35:775-792, 1997.

[67] M. Piatak, M.S. SaAG, L.C. Yang, S.J. Clark, and D. Ho. High levels of HIV1 in plasma during all stages of infection determined by competitive PCR. Science, 259:1749-1754, 1993.

[68] J. Stephenson. New anti-HIV drugs and treatment strategies buoy AIDS researchers. JAMA, 275:579-580, 1996.

[69] G.X. MCLEOD AND S.M. HAmmer. Zidovudine: 5 years later. Annals of Internal Medicine, 117:487-501, 1992.

[70] P.A. Volberding AND ET AL. The duration of zidovudine benefit in persons with asymptomatic HIV infection. JAMA, 272:437-442, 1994.

[71] A.C. Collier, R.W. Coombs, D.A. Schoenfeld, R.L. Bassett, J. Timpone, A. BARUCH, AND ET AL. Treatment of human immunodeficiency virus infection with saquinavir, zidovudine, and zalcitabine. N. Engl. J. Med., 334:1011-1017, 1996 .

[72] J. Cohen. Shooting for the moon with drugs. Science, 273:302, 1996.

[73] W.W. Grody, S. Fligiel, and F. Naeim. Thymus involution in the acquired immunodeficiency syndrome. Am. J. Clin. Pathol., 84:85-95, 1985.

[74] J. Alvarez-Ramirez, M. Meraz, and J.X. Velasco-Hernandez. A feedback control strategy for the chemotherapy of HIV. International J. Of Bif. and Chaos, 10:2207-2220, 2000. 